\title{
Simplified model for fast optimization of a free-electron laser oscillator
}

\author{
Kai Li, ${ }^{1,2}$ Minghao Song, ${ }^{1,2}$ and Haixiao Deng ${ }^{1, *}$ \\ ${ }^{1}$ Shanghai Institute of Applied Physics, Chinese Academy of Sciences, Shanghai 201800, China \\ ${ }^{2}$ University of Chinese Academy of Sciences, Beijing 100049, China
}

(Received 26 October 2016; published 7 March 2017)

\begin{abstract}
A simplified one-dimensional theoretical model for free-electron laser oscillator (FELO) calculation which reserves the main physics is proposed. Instead of using traditional macroparticles sampling method, the theoretical model takes advantage of low gain theory to calculate the optical power single-pass gain in the undulator analytically, and some reasonable approximations are made to simplify the calculation of power growth in the cavity. The theoretical analysis of single-pass gain, power growth, time-dependent laser profile evolution and cavity desynchronism are accomplished more efficiently. We present the results of infrared wavelength FELO and X-ray FELO with the new model. The results are validated by simulation with GENESIS and OPC.
\end{abstract}

DOI: 10.1103/PhysRevAccelBeams.20.030702

\section{INTRODUCTION}

Free-electron laser (FEL) is a new light source which uses relativistic electron beam passes through the undulator and interacts with the radiation field to generate high brilliance laser pulses. Due to its many advantages, such as rapid and continuous tunability over a wide spectral range, lots of interests are attached to it currently. Singlepass high-gain FEL, especially self-amplified spontaneous emission (SASE) scheme, is able to produce high brilliance laser pulses in X-ray region. With the great success of Linear Coherent Light Source [1] and Spring-8 Angstrom Compact Free Electron Laser [2], several hard X-ray SASE FEL have been built or under construction around the world. Although SASE FEL provides fully transversely coherent and short temporal duration X-ray pulses, it starts from shot noise and produces poorly temporal coherent light [3]. Numerous schemes, including external seeded FEL [4-6] and self-seeding [7,8], have been proposed to solve this problem and improve its performances.

Another promising operating mode is free-electron laser oscillator (FELO), which works in the low-gain region and employs electron beam to pass the undulator multiple times to convert energy to radiation. Many long wavelength FELO have been established, e.g., the free-electron laser for infrared experiments [9] and Duke storage-ring based FEL [10]. Also several infrared and THz-FELO are under construction, e.g., the first infrared free-electron laser user facility in China [11]. Recently, a promising schematic X-ray free-electron oscillator (XFELO) [12] has been

\footnotetext{
*denghaixiao@sinap.ac.cn
}

Published by the American Physical Society under the terms of the Creative Commons Attribution 4.0 International license. Further distribution of this work must maintain attribution to the author(s) and the published article's title, journal citation, and DOI. reconsidered thanks to the development of high-reflectivity high-resolution X-ray crystal [13]. XFELO can generate fully temporal coherent and stable peak power laser pulses with the peak brilliance comparable to SASE and the average brilliance several orders of magnitude higher than SASE. Although it holds a lot of potential, there are still some challenges for FELO, including high repetition rate electron injector [14], heat loading of the Bragg reflection crystal mirror [15], and X-ray optics.

In addition, unlike SASE FEL in which electron beam passes undulator once, FELO contains an oscillator in which electron beam and radiation pulse go through undulator hundreds of times before saturation. Thus tracking the electrons motion and radiation field evolution requires lots of calculation and thus the theoretical analysis and design of FELO become another problem. Although there are some conventional FEL simulation codes such as GENESIS [16], GINGER [17], and by combining with optical codes OPC [18] can simulate FELO, these approaches are usually relatively slow and time-consuming. For example, according to [12,19] a complete tracking from the initial spontaneous emission to final saturation of XFELO takes about one month. Thus there is a strong scientific demand for a simpler and faster theoretical model, which is able to obtain some primary results, basic performances, and optimum parameters of XFELO with acceptable accuracy.

Traditional simulation approaches mentioned above use the macroparticles sampling method, which contains tens of thousands of macroparticles in each slice, and tracking all of them is laborious and time consuming. In this paper, we propose a new one-dimensional theoretical model which takes advantages of the electron distribution function to solve the single-pass gain as a function of electric field intensity, and some assumptions are used to simplify the calculation of radiation power in order to save time. 
The new approach reduces the calculation time for a full tracking of FELO from days to minutes by analyzing single-pass gain, power growth, laser profile evolution, and cavity desynchronism in a more efficient way. This paper is arranged as follows: the second section introduces theoretical model. Then we show two examples: $1.6 \mu \mathrm{m}$ infrared wavelength FELO and $1 \AA$ X-ray FELO. Finally a brief conclusion of this paper is given.

\section{THEORETICAL MODEL OF FEL OSCILLATOR}

A FELO facility typically contains multiple mirrors with reflectivity $R$ to form an optical cavity which captures the radiation emitted by relativistic electrons traveling through the undulator. In this paper we focus on the two-mirror optical resonator and other multimirror cases are similar to it. The initial spontaneous radiation emitted by the electron beam passing through the undulator acts as a seed for the following amplifying. With the careful synchronism between electron beam and optical pulse, the radiation starting from the shot noise overlaps with electrons and is amplified on successive passes. The electron beam energy is modulated and converted to radiation pulse in the undulator. In order to ensure the increase of optical power, single-pass gain $g$ should overcome net loss, i.e., $(1+g) R_{\text {total }}>1$. The radiation field evolves in the cavity and for the $(n+1)$ th pass at the entrance of undulator

$$
E_{n+1}(t)=\left[E_{n}(t) g(t)+\delta E(t)\right] R_{\text {total }}
$$

where $\delta E$ is the spontaneous radiation, $g(t)$ is the gain of optical field, and $R_{\text {total }}$ is the equivalent reflectivity of two mirrors. The radiation experiences an exponential growth before the gain begins to drop off due to too large energy spread. It takes about hundreds of passes before the singlepass gain equals the round-trip cavity loss, and then the FELO reaches saturation and remains at constant output power finally.

The main process laser pulse undergoes during one round-trip can be divided into two main parts: the interaction with electron beam in the undulator and the reflections of mirror at the two sides of cavity. In order to start simulations with the new FELO theoretical model using Eq. (1), three elementary procedures that are described by $E_{0}(t), g(t)$ and $R_{\text {total }}$ are analyzed in the following.

\section{A. Initial startup}

The FELO starts from the electron beam synchrotron radiation in the undulator, which is randomly in phase and chaotic in spectrum. This initial shot noise signal acts as a seed which is trapped in the optical cavity and amplified on the successive passes. According to [20] an electron passing through an undulator with $N_{u}$ periods produces a wave train

$$
E_{e}(t)= \begin{cases}A_{0} \exp \left(-i \omega t+\phi_{0}\right) & \text { if }-T / 2<t<T / 2, \\ 0 & \text { otherwise, }\end{cases}
$$

where $T=N_{u} \lambda /(\beta c)$ is the time for electron travel through the undulator. Each coherent length $N_{u} \lambda$ covers $N_{c}(t) \propto$ $I(t)$ electrons and the radiation field is

$$
E_{0}(t)=A_{0} \exp (-i \omega t) \sum_{j=1}^{N_{c}} \exp \left(i \phi_{j}\right)
$$

where $\phi_{j}$ is the initial phase of electric field which is related to the relative location of electron in the bunch. The average field power $P(t) \propto A_{0}^{2}|b|^{2}$ with $b=\sum_{j=1}^{N_{c}} \exp \left(i \phi_{j}\right)$. The dimensionless variable $\xi=b^{2} /\left\langle b^{2}\right\rangle$ obeys the simple exponential probability distribution

$$
p(\xi)=\exp (-\xi)
$$

Here \langle\rangle means ensemble average over coherent length, and $\left\langle b^{2}\right\rangle=N_{c}[21]$.

With the definition of FEL parameter

$$
\rho=\frac{1}{\gamma}\left[\left(\frac{K[J J]}{4 k_{u} \sigma_{b}}\right)^{2} \frac{I}{2 I_{A}}\right]^{1 / 3}
$$

the initial radiation peak power is [22]

$$
P_{i n}=6 \sqrt{\pi} \rho^{2} \frac{P_{b}}{N_{e} \sqrt{\log N_{e} / \rho}}
$$

where $K$ is the dimensionless undulator parameter, $k_{u}$ is the undulator wave number, $\gamma$ is the ratio of the electron kinetic energy to its rest mass energy, $\sigma_{b}$ is the rms value of beam transverse size, $I$ is the peak current, $I_{A} \approx 17 \mathrm{kA}, P_{b}$ is the electron beam power, and $N_{e}=I \lambda / c e$ is the number of electrons per radiation wave length,

$$
[J J]=J_{0}\left(\frac{K^{2}}{4+2 K^{2}}\right)-J_{1}\left(\frac{K^{2}}{4+2 K^{2}}\right)
$$

where $J_{n}$ is the $n$th Bessel function.

In the numerical model, a Gaussian profile electron bunch is chosen. We use Eqs. (3) and (4) to obtain the initial field power distribution $P(t)$ and then extract the square root to find the distribution of initial electric field profile $E_{0}(t)$. The electric field phases are produced by a random generator of uniform distribution among $[0,2 \pi]$. The roundtrip synchrotron radiation $\delta E(t)$ is assumed equal to $E_{0}(t)$.

\section{B. Gain calculation}

Due to its relatively low field amplitude, FEL low-gain theory is suitable for analyzing the increase of optical power. According to the low-gain theory $[23,24]$, the evolution of electron distribution and thus the gain of laser 
power are solved analytically. We derive formulas in onedimensional approximation, and focus on the longitudinal component of laser field and electrons coordinate. The motion of single electron in the phase space $(\theta, \eta)$ is described by the "pendulum equation" [25]

$$
\begin{gathered}
\frac{d \theta}{d z}=2 k_{u} \eta \\
\frac{d \eta}{d z}=-\frac{\epsilon}{2 k_{u} L_{u}^{2}} \sin \theta
\end{gathered}
$$

where $\theta$ is the phase of electron relevant to its relatively longitudinal position inside the bunch, $\eta=\delta \gamma / \gamma$ is the normalized electron energy deviation from resonance and the undulator length $L_{u}$. We also introduce the field strength parameter

$$
\epsilon=\frac{e E_{0} K[J J]}{\gamma^{2} m c^{2}} k_{u} L_{u}^{2} .
$$

The electron distribution function $\rho(z ; \eta, \theta)$ at point $z$ is governed by the continuity equation

$$
\frac{\partial \rho}{\partial z}+\dot{\theta} \frac{\partial \rho}{\partial \theta}+\dot{\eta} \frac{\partial \rho}{\partial \eta}=0
$$

where $\dot{x}=d x / d z$. Substituting Eqs. (5) and (6) into Eq. (7) and using scaled parameters

$$
\begin{gathered}
z^{\prime}=\frac{\sqrt{\epsilon}}{L_{u}} z \\
\eta^{\prime}=\frac{2 k_{u} L_{u}}{\sqrt{\epsilon}} \eta
\end{gathered}
$$

yield the following partial derivation equation

$$
\frac{\partial \rho}{\partial z^{\prime}}+\eta^{\prime} \frac{\partial \rho}{\partial \theta}+\sin \theta \frac{\partial \rho}{\partial \eta^{\prime}}=0
$$

Assuming the initial distribution of electron beam $\rho_{0}$ fulfills Gaussian function with scaled energy spread $\sigma_{\eta^{\prime}}$ and scaled energy deviation $\eta_{0}^{\prime}$, the solution can be found by the method of characteristics at the end of undulator is

$$
\rho\left(z^{\prime} ; \eta^{\prime}, \theta\right)=\frac{1}{2 \pi} \frac{1}{\sqrt{2 \pi} \sigma_{\eta^{\prime}}} \times \exp \left\{-\frac{1}{2 \sigma_{\eta^{\prime}}^{2}}\left[\frac{\eta^{\prime} \operatorname{cn}\left(z^{\prime} ; C\right)-\sin \theta \operatorname{sn}\left(z^{\prime} ; C\right) \operatorname{dn}\left(z^{\prime} ; C\right)}{1-\cos ^{2} \frac{\theta}{2} \operatorname{sn}^{2}\left(z^{\prime} ; C\right)}-\eta_{0}^{\prime}\right]^{2}\right\}
$$

where $C^{2}=\eta^{\prime 2} / 4+\cos ^{2}(\theta / 2)$ and $c n, s n, d n$ are Jacobi elliptic functions. Note that the influence of electron beam emittance $\varepsilon$ can be involved by replacing the electron beam relative energy spread $\sigma_{E} / E_{0}$ with equivalent relative energy spread

$$
\frac{\sigma_{E}^{\prime}}{E_{0}}=\sqrt{\left(\frac{\sigma_{E}}{E_{0}}\right)^{2}+\left(\frac{\varepsilon \lambda_{u}}{4 \lambda \beta_{e}}\right)^{2}}
$$

where $\beta_{e}$ is the average beta function of electron beam.

The power of electron beam energy converted to the radiation is equal to

$$
\Delta P=m_{e} c^{2} \gamma\langle\Delta \eta\rangle \frac{I}{e c \beta} \frac{1}{2 \pi \Sigma^{2}}
$$

where $2 \pi \Sigma^{2}$ is the cross section of electron beam, $\beta$ is the electron velocity scaled by the speed of light $c$, the average change of $\eta$ between the entrance and the end of undulator is

$$
\langle\Delta \eta\rangle=\frac{\sqrt{\epsilon}}{2 k_{u} L_{u}} \int_{0}^{2 \pi} \int_{-\infty}^{+\infty}\left(\rho-\rho_{0}\right) \eta^{\prime} d \eta^{\prime} d \theta
$$

and thus the power gain of the laser is

$$
g=\frac{\Delta P}{P_{0}}
$$

where $P_{0}$ is the initial radiation power. To improve the speed of calculation in the numerical simulation, the singlepass gain $g(t)$ as a function of radiation amplitude $E(t)$ is established as a data base and the gain corresponds to different slices at each round-trip is extracted by linear interpolation.

\section{Cavity mirror reflectivity}

The FELO facility typically contains a mirror at two sides which forms a cavity to trap the optical pulse. For infrared wavelength light, metal mirrors are utilized due to its broadband reflectivity and high thermal conductivity. The interaction between radiation and mirror can be approximated by a normal reflection without spectral modulation, the reflectivity $R_{\text {total }}$ and output-coupling are constant. However, it is more complicated for XFELO which exploits Bragg crystal reflection. The highreflectivity spectral bandwidth for crystal mirror is relatively much narrow so that the optical pulses are cut off in the frequency domain and deformed in the temporal space $[26,27]$. Although the reflectivity of crystal mirror is dependent on lots of factors, such as X-ray incident angle, profile of pulse, and thickness of crystal, to illustrate the 


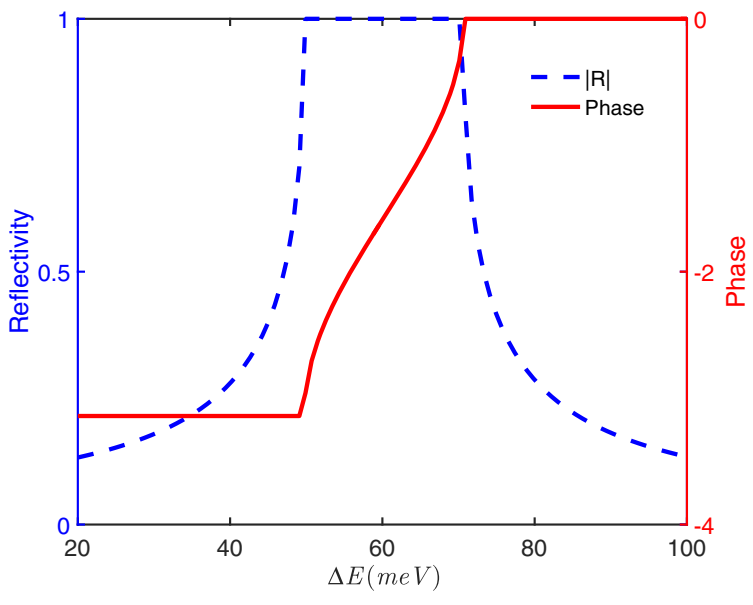

FIG. 1. The complex reflectivity of Bragg crystal at various incident photon energy deviation from Bragg energy.

main properties of Bragg backscattering, we assume the crystal to be semi-infinite and nonabsorbing, and the symmetry Bragg backward scattering is chosen. In this way, the complex reflectivity for the incident photon energy $E$ is simplified as [28]

$$
R(y)=\left\{\begin{array}{cl}
y-\sqrt{y^{2}-1} & \text { if } y>1 \\
y-i \sqrt{1-y^{2}} & \text { if }|y| \leq 1 \\
y+\sqrt{y^{2}-1} & \text { if } y<-1
\end{array}\right.
$$

where $y=\frac{1}{\chi_{H}}\left[\frac{2\left(E-E_{H}\right)}{E_{H}}+\chi_{0}\right], E_{H}$ is the Bragg energy, and $\chi_{0}$ and $\chi_{H}$ are Fourier components of the dielectric susceptibility of the crystal. Figure 1 shows the reflectivity of diamond crystal $\mathrm{C}(4,4,4)$ in symmetry Bragg backscatter at various incident photon energy deviation $\Delta E=E-E_{h}$. The shift of curve central from zero results from the fact that peak reflectivity located at an energy slightly different from the Bragg energy.

TABLE I. Simulation parameters for infrared FELO.

\begin{tabular}{lcr}
\hline \hline Parameter & Value & Unit \\
\hline Beam energy & 80 & $\mathrm{MeV}$ \\
Slice relative energy spread $\sigma_{\eta}$ & $0.2 \%$ & \\
Normalized emittance $\varepsilon_{n}$ & 10 & $\mu \mathrm{m}$-rad \\
Peak current $I$ & 200 & $\mathrm{~A}$ \\
Electron beam charge $Q$ & 100 & $\mathrm{pC}$ \\
Electron bunch length (FWHM) $\sigma_{e}$ & 0.5 & $\mathrm{ps}$ \\
Undulator period $\lambda_{u}$ & 45 & $\mathrm{~mm}$ \\
Number of undulator $N_{u}$ & 16 & \\
Laser wavelength $\lambda$ & 1.6 & $\mu \mathrm{m}$ \\
Rayleigh length $Z_{R}$ & 0.35 & $\mathrm{~m}$ \\
Cavity loss & $1 \%$ & \\
Output coupling efficiency & $6 \%$ & \\
\hline \hline
\end{tabular}

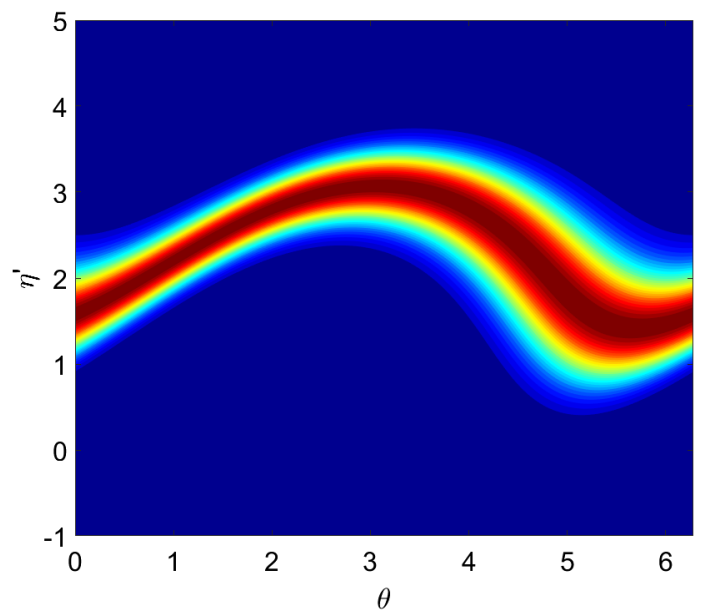

FIG. 2. The electron density distribution function in phase space of one slice.

In the numerical simulation of XFELO, the radiation spectrum is first calculated by Fourier transform, and then multiply by the corresponding reflectivity. Finally, inverse Fourier transform is performed to get the pulse temporal profile after the Bragg reflection.

\section{INFRARED FELO SIMULATION}

A typical infrared FELO is investigated using parameters shown in Table I, which are also presented in [29]. The modulation of electron distribution function in the phase space and the gain degradation as the radiation electric field increases are obtained by Eq. (9). When calculating the gain we assume that the FELO is operated at its optimum beam energy deviation which produces the maximum single-pass gain. And the electron bunch cross section is considered the same as the optical beam waist which is determined by Rayleigh length $Z_{R}$. Figure 2 shows the contour map of electron distribution in the phase space of

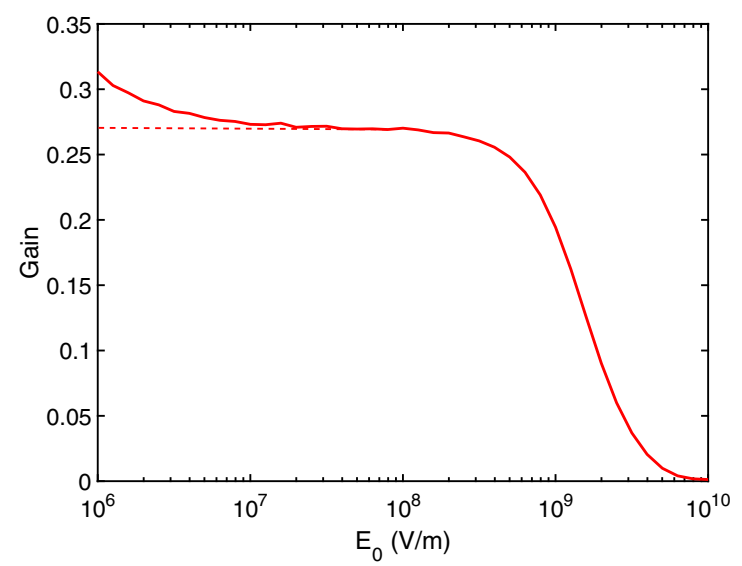

FIG. 3. The gain as a function of electric field intensity. (The dash line is the expected constant gain function curve in the lowgain theory at small electric field intensity.) 


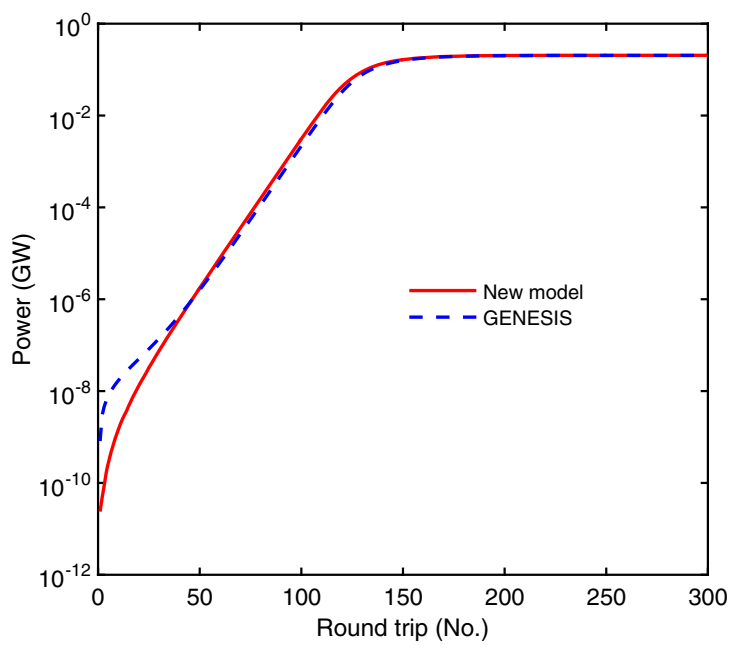

FIG. 4. The enhancement of output laser peak power with various passes $N_{\text {pass }}$.

one slice. As expected, the initial Gaussian profile is twisted and the electrons rotates in the "bucket." which means the electrons energy are modulated and transformed to a laser pulse. As a consequence of energy conservation law, the growth of laser power is equal to the loss energy of electron bunch which can be obtained by difference between initial and final total energy using Eq. (12). The single-pass gain as a function of optical field is given by Fig. 3, it decreases as laser power rises. However, it drops off a little at the beginning which is contradict to the predicted constant gain in low-gain theory. Further investigation reveals that is due to the relative large value of $\eta^{\prime}$ leads to low accuracy of integration of electron density function at the small radiation power. By refine the mesh grids in the integration process, it can be mitigated.
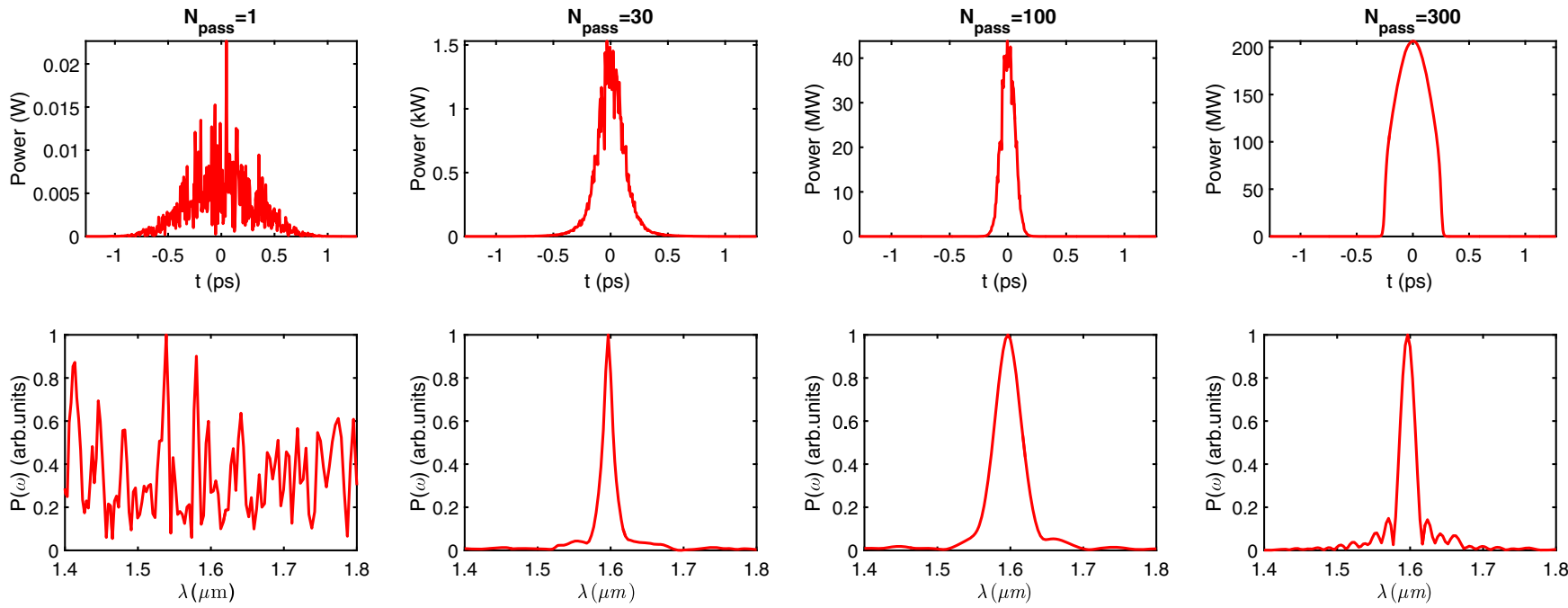

It is worthy to note that, even refining the mesh grids enhances the accuracy of gain calculation, the Madey theorem [30] is used to get the gain at low field amplitude while solving the gain by the new model near the saturation. Low gain perturbation theory requires $\epsilon \ll 1$, thus criticism chosen in our simulation is $\epsilon \leq 0.1$. The specific value of it should be adjusted according to the mesh grids to let the gain curve connect smoothly at the transition point. Through the combination of these two approaches, single-pass gain for all electric field intensity can be given effectively and correctly.

The output coupling of the downstream mirror is $6 \%$ and extra $1 \%$ cavity loss due to the upstream mirror absorbing or diffraction of light is considered. To evaluate the evolution of laser pulse profile and spectrum bandwidth in the oscillator, we use Eq. (3) to get the initial shot noise startup. The growth of laser power is calculated by Eq. (1) until it approaches saturation and remains constant. According to the FEL resonance condition, the electric field propagates ahead of electron beam one resonant wavelength when travel through one undulator period. By the virtue of several hundred times of pass through undulator and continually slippage, the field in different slices "communicate" with each other and develops into a high brilliant, monochromatic laser pulse. Due to the chaotic phase at the beginning, the initial gain is relatively small [31], the influence of slippage is roughly considered as phase averaging of different slices within a coherent length.

Figure 4 displays the growth of laser pulse after various number of pass. The evolution of both spectral and temporal profile of radiation in the cavity from shot noise to saturation are demonstrated in Fig. 5 at the upper and bottom row of panels, respectively. These plots indicate

FIG. 5. Snapshots of output radiation pulse for a typical infrared FELO at $1.6 \mu \mathrm{m}$. The top and the bottom row show the longitudinal pulse temporal profile and corresponding spectrum respectively [33]. 
significant temporal and spectral fluctuations during the initial amplification period and become smooth as passing number increases. The FELO finally generate a 423 fs (FWHM) laser pulse with $23.5 \mathrm{~nm}$ (FWHM) spectral bandwidth at $1.6 \mu \mathrm{m}$. Excepting a little earlier boost of laser power in Fig. 4 and larger of pulse duration, which are due to the roughly treatment of slippage, the results agree well with Ref. [29] both in temporal and spectrum profile as well as peak power intensity. Thus it proves the accuracy of the new theoretical model. In addition, the laser pulse temporal width first narrows and then broadens, which is consistent with the analysis in Ref. [32].

It is interesting to note that since the new theoretical model generates results more efficiently, it can be adjusted to investigate the cavity desynchronism in FELO. Due to slippage, the group velocity of the electric field is smaller than the speed of light, which leads to the maximum gain appears to lag behind and miss out the largest electron density location. This leads to the degradation of output laser peak power [34,35]. It is hard to calculate the group

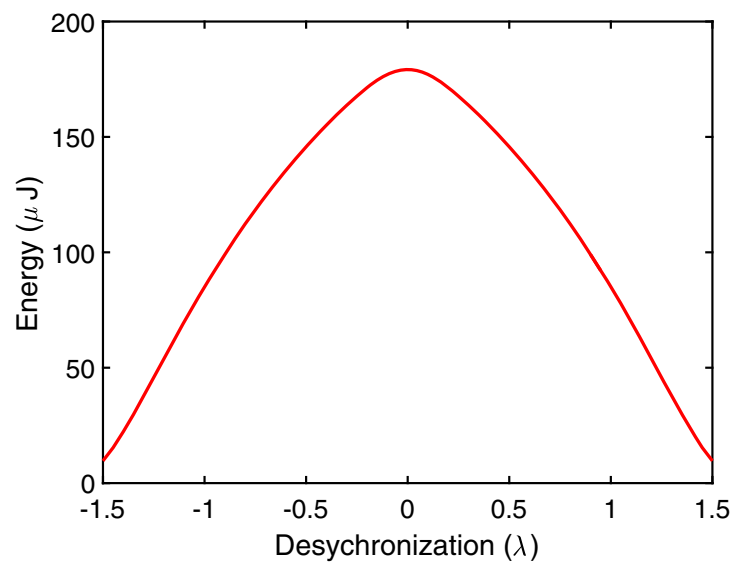

FIG. 6. The output laser energy as a function of desynchronism.

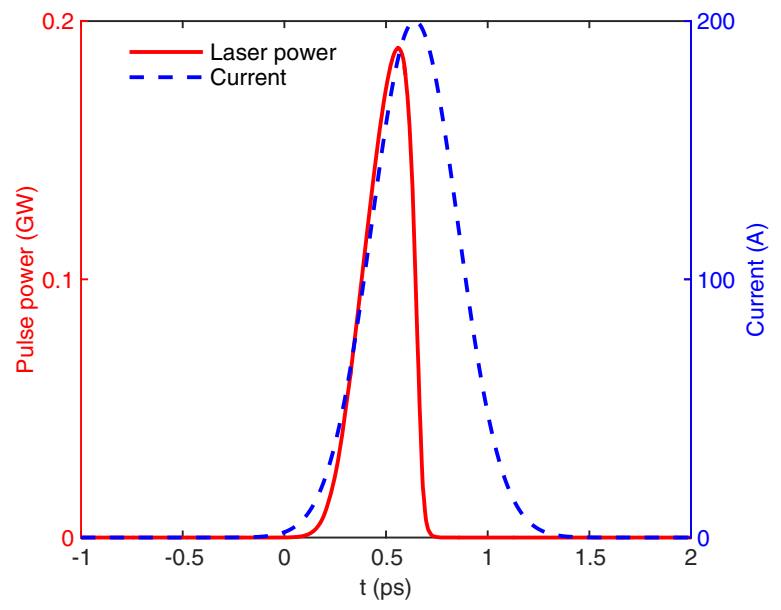

FIG. 7. The laser profile at $0.6 \lambda$ cavity desynchronism. velocity of light precisely in our model, so we ignore the "lethargy" effect and assume the electron bunch and laser pulse already at perfectly synchronism. However, we can deliberately shift the electron profile of a different range and investigate its influence to output laser energy. Figure 6 shows the output energy declines when cavity desynchronism increases. Figure 7 demonstrates a typical light profile at $0.6 \lambda$ cavity desynchronism and it is obvious that the pulse tilts toward electron beam slightly.

\section{X-RAY FELO SIMULATION}

A typical $1 \AA$ X-ray XFELO is investigated with the parameters in Table. II. The optical cavity is built up with diamond crystal mirrors by $\mathrm{C}(4,4,4)$ Bragg reflection with the Bragg energy at $12.04 \mathrm{keV}$ and reflectivity reaches 94\%. The Bragg crystal mirrors filter out the frequency components beyond its high-reflectivity spectral bandwidth nearly $20 \mathrm{meV}$, and in order to maintain enough gain to overcome the round-trip loss and make the radiation power growth, the number of undulator periods is chosen to be 3000. In this case the single-pass gain is near 39\% which is given by the theoretical gain calculation method mentioned above. The electron density distribution in the phase space of a slice, when pulse power inside the cavity equals to $0.2 \mathrm{MW}$, is shown in Fig. 8. Due to the relatively larger electron energy, the bucket traps the electrons becomes flatter, and the energy modulation is smaller. After a shot period of struggling as the initial shot noise, the laser power inside the cavity increases as it goes through the undulator again and again and becomes a stable $0.57 \mathrm{MW}$ laser pulse output at saturation, which is shown in Fig. 9. The final output power from GENESIS simulation presented with dashed line is nearly twice as large as that from the new model, which is mainly due to the inaccurate evaluation of electron beam and laser cross section as well as the coupling factor between them. Given the approximations used in the analysis process, it is easy to understanding this discrepancy.

The XFELO laser power evolution inside the cavity is solved in the same method as for infrared FELO except that

TABLE II. Simulation parameters for X-ray FELO.

\begin{tabular}{lcr}
\hline \hline Parameter & Value & Unit \\
\hline Beam energy & 7 & $\mathrm{GeV}$ \\
Energy spread $\sigma_{E}$ & 1.4 & $\mathrm{MeV}$ \\
Normalized emittance $\varepsilon_{n}$ & 0.2 & $\mu \mathrm{m}-\mathrm{rad}$ \\
Peak current $I$ & 10 & $\mathrm{~A}$ \\
Electron bunch length $\sigma_{t}$ & 1.0 & $\mathrm{ps}$ \\
Undulator period $\lambda_{u}$ & 17.6 & $\mathrm{~mm}$ \\
Number of undulator $N_{u}$ & 3000 & $\AA$ \\
Laser wavelength $\lambda$ & 1.0 & \\
Output coupling & $8 \%$ & \\
Bragg mirror reflectivity $R_{\text {total }}$ & $85 \%$ & \\
\hline \hline
\end{tabular}




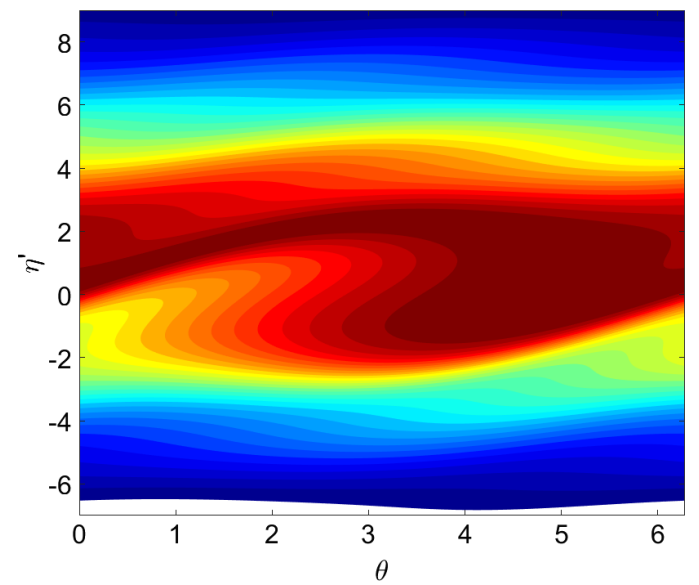

FIG. 8. The electron density distribution in the phase space of one slice.

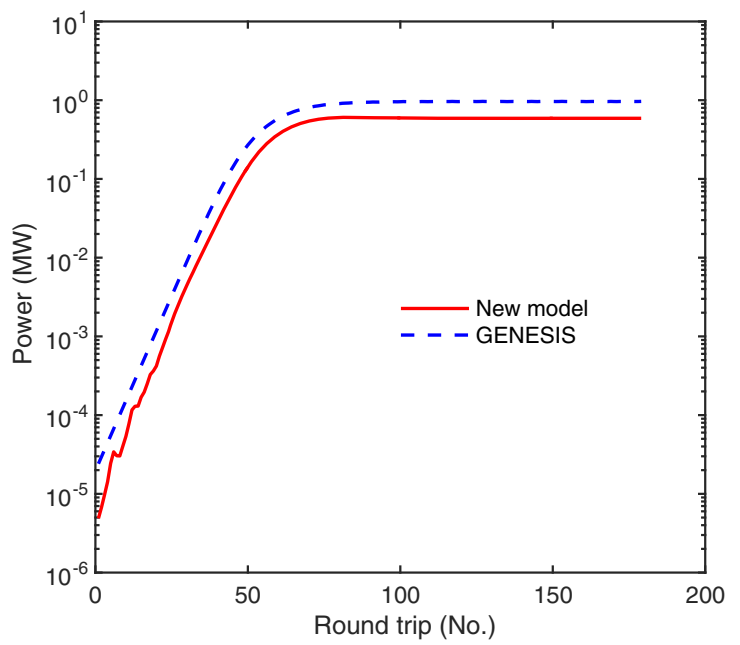

FIG. 9. The enhancement of output laser peak power with various passes $N_{\text {pass }}$.
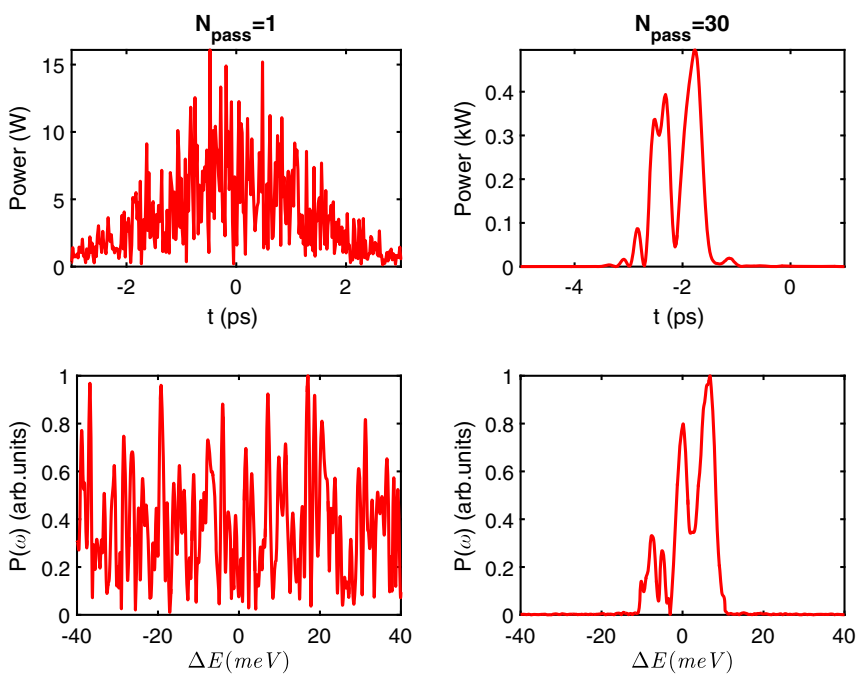

the reflectivity of metal mirrors is replaced by complex reflectivity in Eq. (13) and the snapshots of output pulse profile and the corresponding spectrum are displayed in Fig. 10. The evolution of the laser power and profile are quite similar with the infrared wavelength case. However, besides slippage, the electric field is spectrally narrowed by Bragg crystal mirror reflection heavily for XFELO and this improves the longitudinal coherence of the laser. Finally, as the laser power increases and single-pass gain drops off, FELO transitions to saturation and maintains a steady state.

The complex reflectivity of the crystal mirrors causes an extra phase shift of optical field and leads to the pulse slides backward. The "lethargy" from the crystal mirrors reflection are much larger than that of the infrared FELO, thus cannot be ignored. In the theoretical model, the electron beam is constantly delayed a distance to overlap with the optical field and the shift distance is equal to $20 \mu \mathrm{m}$ to maintain the overlap between electron bunch and laser pulse in Fig. 10. The output laser pulse duration is $1.9 \mathrm{ps}$ (FWHM) with spectral bandwidth is $4.6 \mathrm{meV}$.

The output total energy as a function of this shift distance is demonstrated in Fig. 11. The energy increases sharply when the shift distance larger than $18 \mu \mathrm{m}$ and reduces gradually when it is larger than $50 \mu \mathrm{m}$. The fluctuation of laser energy when the electric field struggles to grow up at edge of over-desynchronism is due to the different initial shot noise at each case. Our result shows that the energy remains constant when the shift distance is $30 \mu \mathrm{m}$ larger than the optimum desynchronism. The flat-top profile of the curve is due the relative narrow highreflectivity spectral bandwidth of crystal mirrors which leads to the corresponding broad temporal pulse profile which acts as a long seed to be amplified. Thus the high intensity output power covers larger desynchronism range in XFELO.
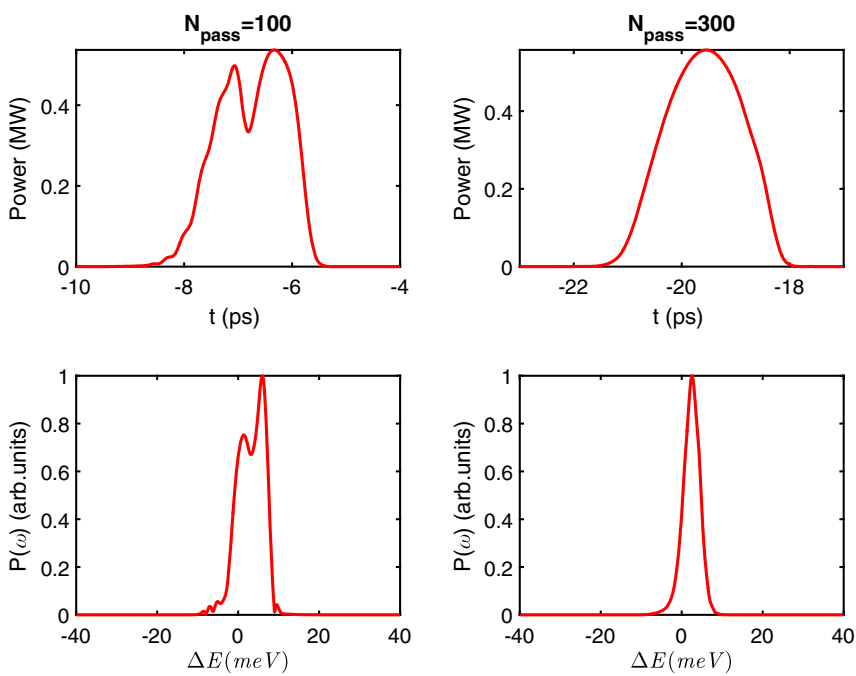

FIG. 10. Snapshots of output radiation pulse for a typical X-ray FELO at $1.0 \AA$. The top and the bottom row show the longitudinal pulse temporal profile and corresponding spectrum respectively [33]. 


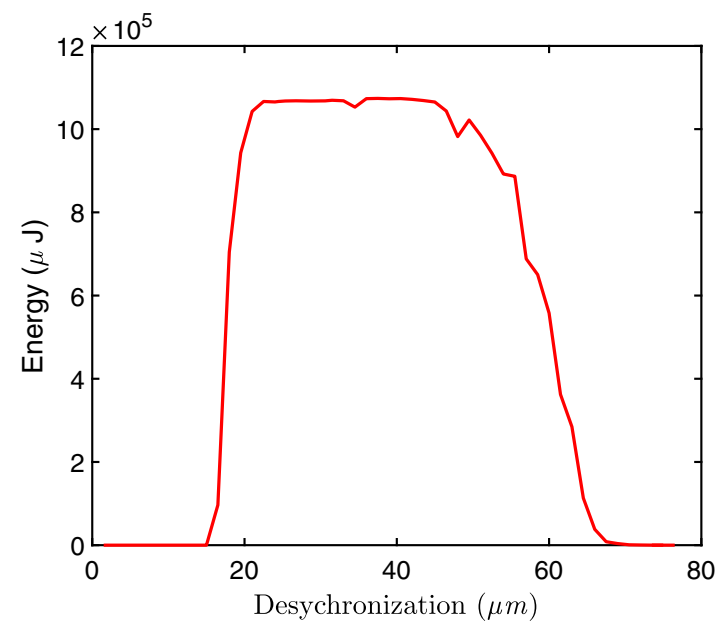

FIG. 11. The output laser energy as a function of electron beam shift distance in each pass.

\section{CONCLUSION}

In this paper we proposed a novel one-dimensional theoretical model which is useful for fast optimization of FELO and obtaining some results in a shorter period of time. The model solved the partial differential equation theoretically in order to obtain the single-pass gain when FELO is approaching saturation. The oscillator mirrors reflection is considered simply by multiplying the corresponding spectral reflectivity. The gain calculation as a separate part takes a few minutes and once the gain function is established as a data base, the oscillator cavity simply calls for the corresponding gain and spends tens of seconds to reaches saturation and returns the needed results.

We investigated the performance of a $1.6 \mu \mathrm{m}$ infrared and a $1 \AA$ X-ray FELO by the new approach. The agreement between our results and those from the GENESIS simulation proves that the new model is feasible and reliable. Taking the advantages of the higher efficiency of the new model, it is easily adjusted to investigate the cavity desynchronism quickly. The electrons initial distribution function is assumed to be Gaussian function, the following work would be to use the truly electron distribution from accelerator tracking to obtain some practical results and to enhance the accuracy of XFELO theoretical model as well as taking into account the influence of laser pulse heating effects on the Bragg crystal mirrors.

\section{ACKNOWLEDGMENTS}

The authors are grateful to Xiaofan Wang, Han Zhang, and Mengying Deng for helpful discussions and useful comments. This work was partially supported by the National Natural Science Foundation of China (11322550) and Ten Thousand Talent Program.
[1] P. Emma, R. Akre, J. Arthur, R. Bionta, C. Bostedt, J. Bozek, A. Brachmann, P. Bucksbaum, R. Coffee, F.-J. Decker et al., Nat. Photonics 4, 641 (2010).

[2] T. Ishikawa, H. Aoyagi, T. Asaka, Y. Asano, N. Azumi, T. Bizen, H. Ego, K. Fukami, T. Fukui, Y. Furukawa et al., Nat. Photonics 6, 540 (2012).

[3] K.-J. Kim, Phys. Rev. Lett. 57, 1871 (1986).

[4] L. H. Yu, Phys. Rev. A 44, 5178 (1991).

[5] D. Xiang and G. Stupakov, Phys. Rev. ST Accel. Beams 12, 030702 (2009).

[6] C. Feng, H. Deng, D. Wang, and Z. Zhao, New J. Phys. 16, 043021 (2014).

[7] J. Amann, W. Berg, V. Blank, F.-J. Decker, Y. Ding, P. Emma, Y. Feng, J. Frisch, D. Fritz, J. Hastings et al., Nat. Photonics 6, 693 (2012).

[8] G. Geloni, V. Kocharyan, and E. Saldin, J. Mod. Opt. 58, 1391 (2011)

[9] D. Oepts, A. Van der Meer, and P. Van Amersfoort, Infrared Phys. Technol. 36, 297 (1995).

[10] J. Yan, H. Hao, J. Li, S. Mikhailov, V. Popov, N. Vinokurov, S. Huang, J. Wu, S. Günster, and Y. Wu, Phys. Rev. Accel. Beams 19, 070701 (2016).

[11] H.-T. Li, Q.-K. Jia, S.-C. Zhang, L. Wang, and Y.-L. Yang, arXiv:1609.01012.

[12] K.-J. Kim, Y. Shvydko, and S. Reiche, Phys. Rev. Lett. 100, 244802 (2008).

[13] Y. V. Shvydko, S. Stoupin, A. Cunsolo, A. H. Said, and X. Huang, Nat. Phys. 6, 196 (2010).

[14] P. Ostroumov, K. Kim, and P. Piot, in Proceedings of LINAC08, Victoria, BC, Canada, Vol. 8 (2008) p. 676.

[15] M.-Q. Song, Q.-M. Zhang, Y.-H. Guo, K. Li, and H.-X. Deng, Chin. Phys. C 40, 048101 (2016).

[16] S. Reiche, Nucl. Instrum. Methods Phys. Res., Sect. A 429, 243 (1999).

[17] W. Fawley, Ginger FEL simulation code, LBNL Technical Report No. 49625, 2001.

[18] P. van der Slot, H. Freund, W. Miner Jr, S. Benson, M. Shinn, and K.-J. Boller, Phys. Rev. Lett. 102, 244802 (2009).

[19] J. Dai, H. Deng, and Z. Dai, Phys. Rev. Lett. 108, 034802 (2012).

[20] P. Schmüser, M. Dohlus, J. Rossbach, and C. Behrens, Free-electron lasers in the ultraviolet and $X$-ray regime (Springer International Publishing, Hamburg, Germany, 2014), Vol. 258.

[21] E. L. Saldin, E. A. Schneidmiller, and M. Yurkov, Opt. Commun. 148, 383 (1998).

[22] S. Reiche, GENESIS1.3 User Manual (2004).

[23] I. Boscolo, M. Leo, R. A. Leo, G. Soliani, and V. Stagno, IEEE J. Quantum Electron. 18, 1957 (1982).

[24] I. Boscolo, M. Leo, R. Leo, G. Soliani, V. Stagno, and C. Pellegrini, J. Appl. Phys. 57, 18 (1985).

[25] Z. Huang and K.-J. Kim, Phys. Rev. ST Accel. Beams 10, 034801 (2007).

[26] Y. Shvydko and R. Lindberg, Phys. Rev. ST Accel. Beams 15, 100702 (2012).

[27] R. R. Lindberg and Y. V. Shvydko, Phys. Rev. ST Accel. Beams 15, 050706 (2012).

[28] R. Lindberg, K.-J. Kim, Y. Shvydko, and W. Fawley, Phys. Rev. ST Accel. Beams 14, 010701 (2011). 
[29] H. Deng, arXiv:1605.00872.

[30] J. Madey, Nuovo Cimento Soc. Ital. Fis. 50B, 64 (1979).

[31] C. Feng, H. Deng, G. Wang, D. Wang, Z. Zhao, and D. Xiang, Phys. Rev. ST Accel. Beams 16, 060705 (2013).

[32] K.-J. Kim, Phys. Rev. Lett. 66, 2746 (1991).

[33] See Supplemental Material at http://link.aps.org/ supplemental/10.1103/PhysRevAccelBeams.20.030702 for the animation of infrared FELO with $0.4 \lambda$ detune, and the animation of XFELO with $45 \mu \mathrm{m}$ detune.

[34] R. Bakker, D. Jaroszynski, A. Van der Meer, D. Oepts, and P. van Amersfoort, IEEE J. Quantum Electron. 30, 1635 (1994).

[35] G. Knippels, R. Mols, A. Van der Meer, D. Oepts, and P. Van Amersfoort, Phys. Rev. Lett. 75, 1755 (1995). 